\title{
A SYSTEM OF FUNCTIONAL-DIFFERENTIAL EQUATIONS ASSOCIATED WITH THE OPTIMAL DETECTION PROBLEM FOR JUMP-TIMES OF A POISSON PROCESS
}

\author{
DONCHO S. DONCHEV \\ Higher Institute of Food and Flavor Industries \\ Department of Mathematics 4000 Plovdiv, Bulgaria
}

(Received March, 1996; Revised December, 1996)

\begin{abstract}
The value function in the optimal detection problem for jump-times of a Poisson process satisfies a special system of functional-differential equations. In this paper, we investigate the system and prove the existence and uniqueness of its solution.
\end{abstract}

Key words: Functional-Differential Equations with Delay, Fundamental Functions, Global Solutions.

AMS subject classifications: $60 \mathrm{G} 99,60 \mathrm{H} 99$.

\section{Introduction}

This system appears in the form of a Bellman equation for the value function of the problem of optimal detection of jump-times of a Poisson process investigated by Donchev [2]. This problem is a generalization of the classical Poisson disorder problem (Shiryaev [5], Davis [1], and Wickwire [6]) which is the following. Let $\left\{z_{t}\right\}$ be a Poisson process whose rate $\rho$ changes from $\lambda$ to $\mu<\lambda$ at a random time $\theta$. The time $\theta$ is zero with probability $x$ and if $\theta>0$, then it is exponentially distributed with parameter $a$. The Poisson disorder problem is to find a way of using only observations on $\left\{z_{t}\right\}$ to predict the value of $\theta$, and to minimize some cost functional depending on the difference between $\theta$ and its predicted value.

In this problem, the disorder occurs only once. Now, we suppose that a multiple Poisson change of the rate of $\left\{z_{t}\right\}$ occurs. We assume that this change takes place at the jump-times $\theta_{n}, n \geq 1$ of some non-observed Poisson process $\left\{y_{t}\right\}$ and that for the rate of $\left\{z_{t}\right\}, \rho=\lambda$ if $y_{t}$ is odd and $\rho=\mu$ if $y_{t}$ is even. The rate of $\left\{y_{t}\right\}$ is equal to $a$ and the apriori probability $x=P\left\{y_{0} \in\{2 n+1, n \geq 0\}\right\}$ is given. A policy is any nondecreasing sequence of stopping times $\left\{\tau_{n}\right\}, n \geq 1$, w.r.t. the filtration generated by the process $\left\{z_{t}\right\}$. The problem is to minimize some cost functional depending on all $\theta_{n}$ and $\tau_{n}, \quad n \geq 1$. Referring the reader for more details to Donchev [2], let us return to the system. It is the following: 


$$
\begin{aligned}
f_{1}^{\prime}(t) & =(\epsilon-2 a \sinh t)^{-1}\left\{\lambda\left[f_{1}(t)-f_{1}(t-\gamma)\right]\right. \\
+ & \left.\left(a+\beta-a e^{t}\right) f_{1}(t)+1\right\}, \quad t \geq 0 \\
f_{2}^{\prime}(t)= & (\epsilon-2 a \sinh t)^{-1}\left\{\lambda\left[f_{2}(t)-f_{2}(t-\gamma)\right]\right. \\
+ & \left.\left(a+\beta-a e^{-t}\right) f_{2}(t)+1\right\}, \quad t \leq 0 \\
& f_{1}(t)=e^{t} f_{2}(t), \quad t \in[-\gamma, 0]
\end{aligned}
$$

where $\mu, \lambda, \epsilon, \beta, \gamma$ and $a$ are positive numbers such that

$$
\lambda>\mu, \epsilon=\lambda-\mu, \gamma=\ln \lambda-\ln \mu .
$$

It follows by dynamic programming reasoning that system (1.1)-(1.3) has a unique solution which coincides with the value function of the above problem. On the other hand, from the point of view of the theory of functional-differential equations such an existence-uniqueness result is not trivial. In particular, if we try to solve system (1.1)-(1.3), there would arise at least two difficulties to overcome. The first is the singularity that equation (1.1) has a point $t=\ln \left(\epsilon / 2 a+\sqrt{1+(\epsilon / 2 a)^{2}}\right)$, and the second is the delaying argument of equation (1.2) on the negative half-line.

Here we prove the existence and uniqueness of the solution of system (1.1)-(1.3) utilizing methods and ideas from the theory of functional-differential equations with delay. This solution cannot be represented by means of known functions. Nevertheless, we describe its structure and obtain results permitting us to develop numerical tools for its calculation.

This article consists of four sections. In Section 2 we investigate the asymptotics of the solutions of equation (1.2) as $t \rightarrow-\infty$ and obtain explicit formulas for the global solutions of both equations (1.1) and (1.2). Section 3 is devoted to the fundamental functions of equations (1.1) and (1.2) and their properties. In Section 4 we prove that the homogeneous equation corresponding to (1.2) has a non-trivial solution on the negative half-line and that the general solution of this equation is a sum of its global solution and the solution of the homogeneous equation multiplied by a constant. In the end of the section we utilize this fact when constructing a solution of the system (1.1)-(1.3).

\section{Asymptotics and Global Solutions}

Equation (1.2) plays an important role in the system (1.1)-(1.3). Further we shall consider also the corresponding homogeneous equation:

$$
\eta^{\prime}(t)=(\epsilon-2 a \sinh t)^{-1}\left\{\mu[\eta(t)-\eta(t=\gamma)]+\left(a+\beta-a e^{-t}\right) \eta(t)\right\}, t \leq 0 .
$$

In Section 4, we show that equation (2.1) has a non-trivial solution on the negative half-line. The next theorem contains aprior information about the asymptotic behavior of this solution.

Theorem 2.1: (i) Every solution of equation (2.1) has the following asymptotic as $t \rightarrow-\infty$ :

$$
\eta(t)=C\left(1+\beta / a+e^{-t}\right)+o(1), C=\text { const. }
$$


(ii) If equation (1.2) has a solution, then this solution also has the asymptotics (2.2) with some constant $C$.

Proof: Let us consider the equation

$$
\begin{gathered}
\{-\epsilon x(1-x)+a(1-2 x)] v^{\prime}(x)+[\lambda x+\mu(1-x)] \\
\times\left[v\left(\frac{\lambda x}{\lambda x+\mu(1-x)}\right)-v(x)\right]-(1-x)=\beta v(x), x \in[1 / 2,1] .
\end{gathered}
$$

As shown by Dontchev [2], this equation reduces to (1.2) with $\gamma=\ln \lambda-\ln \mu$ if one introduces a logarithmic scale $t=\ln (1-x)-\ln x$ and a new unknown function $U(t)=v\left(\left(1+e^{t}\right)^{-1}\right)$ and defines thereafter the function $f_{2}(t)$ by the formula

$$
f_{2}(t)=\left(1+e^{-t}\right) U(t) \text {. }
$$

Moreover, in this case, the homogeneous equation corresponding to (2.3) reduces to (2.1). Thus, if equation (1.2) (resp. equation (2.1)) has a solution, then equation (2.3) (resp. the homogeneous equation corresponding to (2.3)) has a solution as well.

Let $f_{2}(t)$ be a solution of (1.2) and consider the corresponding function $v(x)$. Applying the Lagrange formula to the difference $v\left(\frac{\lambda x}{\lambda x+\mu(1-x)}\right)-v(x)$ in the lefthand side of (2.3) we obtain

$$
v\left(\frac{\lambda x}{\lambda x+\mu(1-x)}\right)-v(x)=v^{\prime}(\xi) \frac{\epsilon x(1-x)}{\lambda x+\mu(1-x)}, \xi \in\left[x, \frac{\lambda x}{\lambda x+\mu(1-x)}\right] .
$$

Substituting this expression into (2.3), we get

$$
-(1-x)+\epsilon x(1-x)\left[v^{\prime}(\xi)-v^{\prime}(x)\right]+a(1-2 x) v^{\prime}(x)=\beta v(x) .
$$

Solving equation (2.3) with respect to $v^{\prime}(x)$ and taking into consideration the facts that $a>0$ and $v(x)$ is a continuously differentiable function, we easily deduce that $v$ obeys a smooth second derivative in a neighborhood of the point $x=1$. Therefore, we can apply the Lagrange formula once again to the difference $v^{\prime}(\xi)-v^{\prime}(x)$ which appears in the second term of (2.5). Thus, we obtain the following equation:

$$
a(1-2 x) v^{\prime}(x)=\beta v(x)+1-x+O\left((1-x)^{2}\right), x \rightarrow 1 .
$$

Let us now return to the logarithmic scale $t=\ln (1-x)-\ln x$ and the function $U(t)$. Then equation (2.6) takes the following form:

$$
2 a U^{\prime}(t) \sinh t=-\beta U(t)-\left(1+e^{-t}\right)^{-1}+O\left(\left(1+e^{-t}\right)^{-2}\right), t \rightarrow-\infty .
$$

The solution of the last equation is

$$
\begin{aligned}
& U(t)= \exp \left(-\frac{\beta}{2 a} \int_{-\infty}^{t} \frac{d u}{\sinh u}\right)\left[C_{1}-\int_{-\infty}^{t} \frac{\exp \left(\frac{\beta}{2 a} \int_{-\infty}^{u} \frac{d s}{\sinh s}\right)}{2 a\left(1+e^{-u}\right) \sinh u} d u\right. \\
&\left.+\int_{-\infty}^{t} \exp \left(\frac{\beta}{2 a} \int_{-\infty}^{u} \frac{d s}{\sinh s}\right) O\left(\left(1+e^{-u}\right)^{-2}\right) \frac{d u}{\sinh u}\right] \\
&=\left(\frac{e^{-t}-1}{e^{-t}+1}\right)^{-\frac{\beta}{2 a}}\left[C_{1}+a^{-1} \int_{-\infty}^{t}\left(e^{-u}-1\right)^{\frac{\beta}{2 a}-1}\left(e^{-u}+1\right)^{-\frac{\beta}{2 a}-2} e^{-u} d u\right.
\end{aligned}
$$




$$
\begin{gathered}
\left.+\int_{-\infty}^{t}\left(\frac{e^{-u}-1}{e^{-u}+1}\right)^{\frac{\beta}{2 a}} \frac{e^{-u} O\left(\left(1+e^{-u}\right)^{-2}\right)}{\left(e^{-u}-1\right)\left(e^{-u}+1\right)} d u\right] \\
C_{1}=\lim _{t \rightarrow-\infty} U(t), \quad t \rightarrow-\infty .
\end{gathered}
$$

The first integral in $(2.8)$ is equal to $\beta^{-1}(2+\beta / a)^{-1}\left\{1-\left(\frac{e^{-t}-1}{e^{-t}+1}\right)^{\frac{\beta}{2 a}}[1+\right.$ $\left.\left.(\beta / a)\left(1+e^{-t}\right)^{-1}\right]\right\}$. In view of the inequalities $\frac{e^{-u}-1}{e^{-u}+1}<1$ and $\frac{1}{e^{-u}-1}<\frac{2}{e^{-u}+1}$ which hold for $u<0$, the second integral can be written out as $\int_{-\infty}^{t}(1+$ $\left.e^{-u}\right)^{-2} O\left(\left(1+e^{-u}\right)^{-2}\right) d\left(1+e^{-u}\right)=O\left(\left(1+e^{-u}\right)^{-3}\right)$. Substituting these expressions in (2.8), we obtain the following asymptotic formula for the function $U(t)$ :

$$
U(t)=C \zeta_{1}(t)+\zeta_{2}(t)+O\left(\left(1+e^{-t}\right)^{-3}\right), \quad t \rightarrow-\infty,
$$

where $\quad \zeta_{1}(t)=\left(\frac{e^{-t}-1}{e^{-t}+1}\right)^{-\frac{\beta}{2 a}}, \quad \zeta_{2}(t)=-\frac{1+(\beta / a)\left(1+e^{-t}\right)^{-1}}{\beta(2+\beta / a)} \quad$ and $\quad C=C_{1}+$ $\beta^{-1}(2+\beta / a)^{-1}$. Applying Newton's formula to the function $\zeta_{1}(t)$ we get

$$
\begin{gathered}
\zeta_{1}(t)=\left(\frac{e^{-t}-1}{e^{-t}+1}\right)^{-\frac{\beta}{2 a}}=\left(1-\frac{2}{1+e^{-t}}\right)^{-\frac{\beta}{2 a}} \\
=1+(\beta / a)\left(1+e^{-t}\right)^{-1}+o\left(\left(1+e^{-t}\right)^{-1}\right), t \rightarrow-\infty .
\end{gathered}
$$

It follows from (2.9) and (2.10) that

$$
U(t)=C_{1}\left[1+(\beta / a)\left(1+e^{-t}\right)^{-1}\right]+o\left(\left(1+e^{-t}\right)^{-1}\right), \quad t \rightarrow-\infty .
$$

Let us note that the function $\zeta_{1}(t)$ is a solution of the homogeneous equation corresponding to (2.7) which appears in place of (2.7) if we consider equation (2.1) and the homogeneous equation corresponding to (2.3) rather than equations (1.2) and (2.3). Thus, both claims of the theorem follow from $(2.4),(2.10),(2.11)$ and the remark after formula (2.4).

As follows from (2.11), the function $\zeta_{2}(t)$ plays an important role in formula (2.9). Namely, it ensures the fulfillment of the identity $C_{1}=\lim _{t \rightarrow-\infty} U(t)$ in (2.11). It is remarkable that the function $f_{2}(t)=\left(1+e^{-t}\right) \zeta_{2}(t)$ is a global solution (i.e., on the whole real line) of equation (1.2).

Theorem 2.2: The functions

$$
\begin{gathered}
f_{1}(t)=-\frac{1+\beta / a+e^{t}}{\beta(2+\beta / a)} \\
f_{2}(t)=-\frac{1+\beta / a+e^{-t}}{\beta(2+\beta / a)}
\end{gathered}
$$

are global solutions of equations (1.1) and (1.2), respectively.

To prove this theorem, it is enough to substitute $f_{1}(t)$ and $f_{2}(t)$ from $(2.12)$ and 
(2.13) into (1.1) and (1.2) and make simple calculations.

Let us note that the functions from (2.12) and (2.13) do not satisfy system (1.1)(1.3) since the condition (1.3) in this case is not satisfied. On the other hand, we could multiply the right-hand side of (2.13) by $e^{t}$ and try to solve equation (1.1) on $[0,-\infty]$ with an initial condition $-\frac{1+(1+\beta / a) e^{t}}{\beta(2+\beta / a)}$ given on $[-\gamma, 0]$. However, in this case we will not be able to escape the singularity that (1.1) has at point $t=$ $\ln \left(\epsilon / 2 a+\sqrt{1+(\epsilon / 2)^{2}}\right.$. To overcome these difficulties, we need a full characterization of the set of all solutions to equation (1.2) on the negative half-line.

\section{Fundamental Functions and Their Properties}

Denote by $V(t, s)$ and $U(t, s)$, the fundamental functions of equations (1.1) and (1.2), respectively. Thus, for any fixed $s, V(t, s)$ (resp. $U(t, s)$ ) is a solution of the homogeneous equation corresponding to (1.1) (resp. (1.2)) with an initial condition given on $[s-\gamma, s]$ by the formula $\varphi(t)=0$ if $s-\gamma \leq t<s$ and $\varphi(t)=1$ if $t=s$. $V(s, s)=$ $U(s, s)=1$ and $V(t, s)=U(t, s)=0$ for $t \leq s$. If $0 \leq t-s \leq \gamma$, then in order to calculate $V(t, s)$ (resp. $U(t, s)$ ) one has to solve the following ordinary differential equation: $f^{\prime}(t)=(\epsilon-2 a \sinh t)^{-1}\left(\lambda+a+\beta-a e^{t}\right) f(t) \quad\left(\right.$ resp. $\quad f^{\prime}(t)=(\epsilon-2 a \sinh t)^{-1}(\mu+a+$ $\left.\left.\beta-a e^{-t}\right) f(t)\right), \quad s \leq t \leq s+\gamma$, with an initial condition $f(s)=1$. Solving these equations, we obtain the following expressions for the functions $V(t, s)$ and $U(t, s)$ :

$$
\begin{gathered}
V(t, s)=\left(\frac{1+x_{2} e^{s}}{1+x_{2} e^{t}}\right)^{b}\left(\frac{1+x_{1} e^{s}}{1+x_{1} e^{t}}\right)^{c} \\
U(t, s)=e^{s-t}\left(\frac{1+x_{2} e^{s}}{1+x_{2} e^{t}}\right)^{b}\left(\frac{1+x_{1} e^{s}}{1+x_{1} e^{t}}\right)^{c}
\end{gathered}
$$

where $x_{1,2}=\epsilon / 2 a \pm \sqrt{1+(\epsilon / 2 a)^{2}}$ are the roots of the equation $x^{2}-(\epsilon / a) x-1=0$,

$$
b=\frac{1+(\mu+\beta) / a+x_{2}}{x_{1}-x_{2}}, c=-\frac{1+(\mu+\beta) / a+x_{1}}{x_{1}-x_{2}} .
$$

It is easy to verify that $b>0$ and that the identity $b+c=-1$ holds. It follows from (3.1) and (3.2) that

$$
U(t, s)=e^{s-t} V(t, s), \quad s \leq t \leq s+\gamma .
$$

Since $U(t, s)=V(t, s)=0$ if $t<s,(3.4)$ is obviously fulfilled also for all $t<s$. If $t>s+\gamma$, then the formulas for $V(t, s)$ and $U(t, s)$ are more complicated. Nevertheless, it turns out that formula (3.4) holds in this case as well.

Consider the equations:

$$
\begin{gathered}
\left.f^{\prime}(t)=(\epsilon-2 a \sinh t)^{-1}\left\{\lambda[f(t)-f(t-\gamma)]+\left(a+\beta-a e^{t}\right) f(t)\right)\right\} \\
f^{\prime}(t)=(\epsilon-2 a \sinh t)^{-1}\left\{\mu[f(t)-f(t-\gamma)]+\left(a+\beta-a e^{-t}\right) f(t)\right\},
\end{gathered}
$$

and denote by $f_{1}(\sigma, \varphi)(t)$ (resp. $\left.f_{2}(\sigma, \varphi)(t)\right), \sigma \in \mathbb{R}, \varphi \in C([\sigma-\gamma, \sigma]$ ), the solution of equation (3.5) (resp. equation (3.6)) on $[\sigma, \infty)$ with an initial condition $\varphi$.

Theorem 3.1: (i) For every real $\sigma$ and $\varphi \in C([\sigma=\gamma, \sigma])$, 


$$
f_{1}\left(\sigma, e^{(\cdot)} \varphi\right)(t)=e^{t} f_{2}(\sigma, \varphi)(t), t \geq \sigma
$$

(ii) The identity (3.4) is satisfied for all real $t$ and $s$.

Proof: Making use of Hale ([4], Theorem 6.3.2), we obtain the following representation for the functions $f_{1}\left(\sigma, e^{(\cdot)} \varphi\right)(t)$ and $f_{2}(\sigma, \varphi)(t)$ for $t \geq \sigma$ :

$$
\begin{aligned}
f_{2}(\sigma, \varphi)(t)=U(t, \sigma) \varphi(\sigma) & \\
& -\mu \int_{\sigma-\gamma}^{\sigma} \varphi(s) U(t, s+\gamma)[\epsilon-2 a \sinh (s+\gamma)]^{-1} d s \\
f_{1}\left(\sigma, e^{(\cdot)} \varphi\right)(t) & =V(t, \sigma) e^{u} \varphi(\sigma) \\
& -\lambda \int_{\sigma-\gamma}^{\sigma} \varphi(s) e^{s} V(t, s+\gamma)[\epsilon-2 a \sinh (s+\gamma)]^{-1} d s .
\end{aligned}
$$

First, we shall prove the claim $(i)$ in case $\sigma \leq t \leq \sigma+\gamma$. Since $t \leq s+2 \gamma$ for all $s \in[\sigma-\gamma, \sigma]$ one can apply formula (3.4) to both $V(\bar{t}, \sigma)$ and $V(t, s+\bar{\gamma})$ in the righthand side of (3.9). Making simple calculations, we get (3.7).

Now we are in a position to prove $(i i)$. Let us fix $s$ and divide the interval $[s, \infty)$ into segments of length $\gamma:[s, \infty)=\bigcup_{n>0}[s+n \gamma, s+(n+1) \gamma)$. We shall prove by induction that (3.4) holds in every segment $[s+n \gamma, s+(n+1) \gamma), n=0,1, \ldots$ For $n=0$, the claim was already proved. Assuming that (ii) holds true for $t \in[s+k \gamma$, $s+(k+1) \gamma)$ and some integer $k \geq 0$ we shall prove that (3.4) is satisfied in the next segment as well. Indeed, to find the functions $V(t, s)$ and $U(t, s)$ on $[s+(k+1) \gamma$, $s+(k+2) \gamma)$ one must solve equations (3.5) and (3.6), respectively, taking as initial conditions the already calculated values of these functions on $[s+k \gamma, s+(k+1 \gamma)$. According to the induction hypothesis, (3.4) holds on $[s+k \gamma, s+(k+1) \gamma)$. Applying (i) with $\sigma=s+(k+1) \gamma, \varphi(\cdot)=e^{-s} U(\cdot, s)$ and $t \in[s+(k+1) \gamma, s+(k+2) \gamma)$ we get the claim for $n=k+1$. Thus, $(i i)$ is proved.

In order to prove (3.7) for $\sigma \leq t \leq \sigma+\gamma$, we have used only formula (3.4) with $t-s \leq \gamma$. Since, according to $(i i)$, the last formula holds for all $t$ and $s$ it follows that (3.7) is fulfilled for all $t \geq \sigma$.

Consider the following domain in the plane $(s, t): D=\{(s, t): s \leq t \leq 0\}$.

Theorem 3.2: The function $U(t, s)$ is continuous and bounded in $\bar{D}$.

Proof: The coefficients of equation (3.6) are bounded continuous functions provided $t \geq 0$. For any fixed $s \leq 0$, function $U(t, s)$ is given by formula (3.2) if $s \leq t \leq s+$ $\gamma$ and it satisfies equation (3.6) with an initial condition $f_{\sigma+\gamma}(\cdot)=U_{s+\gamma}(\cdot, s)$ if $t \geq s+\gamma$. Here, as is generally assumed in the theory of functional-differential equations, for any $f \in C([s, t)), t \geq s+\gamma$ and $\tau \in[s+\gamma, t], f_{\tau}(\cdot)$ denotes a function belonging to $C([-\gamma, 0])$ which is defined by the formula $f_{\tau}(\theta)=f(\tau+\theta), \theta \in$ $[-\gamma, 0]$. In view of $(3.2)$, since $s+\gamma+\theta \in[s, s+\gamma]$ provided $\theta \in[-\gamma, 0]$, it follows that

$$
\begin{gathered}
U_{s+\gamma}(\theta, s)=e^{-\theta-\gamma}\left(\frac{1+x_{2} e^{s}}{1+x_{2} e^{s+\gamma+\theta}}\right)^{b}\left(\frac{1+x_{1} e^{s}}{1+x_{1} e^{s+\gamma+\theta}}\right)^{c}, \\
\theta \in[-\gamma, 0] .
\end{gathered}
$$

The last function is continuous in both $\theta$ and $s$ if $\theta \in[-\gamma, 0]$ and $s \leq-\gamma$. Being 
a solution of equation (3.6) with the continuous initial condition (3.10), the function $U(t, s)$ is continuous w.r.t. $t$ if $t \geq s+\gamma$. Its continuity if $t \in[s, s+\gamma]$ follows from formula (3.2). It remains to be proved that $U(t, s)$ is continuous w.r.t. $s$ for every fixed $t \leq 0$. The proof is non-trivial only if $s<t-\gamma$. Let $s<t-\gamma$ and $\left\{x_{n}\right\}$ be a sequence converging to $s$. Then, because of the continuity of function (3.10) w.r.t. both $\theta$ and $s$, the corresponding sequence of initial conditions $\left\{U_{s_{n}+\gamma}\left(\cdot, s_{n}\right)\right\}$ will converge uniformly to $U_{s+\gamma}(\cdot, s)$. Thus, the continuity of $U(t, s)$ in $s$ follows from the theorem for the continuous dependence of the solutions of functional-differential equations to the initial data (Hale [4], Theorem 2.2.2).

In order to prove the second claim of the theorem, let us rewrite the equation (3.6) in the form

$$
f^{\prime}(t)=-A(t) f(t)-B(t) f(t-\gamma)
$$

where $A(t)=(\epsilon-2 a \sinh t)^{-1}\left(a e^{-t}-a-\mu-\beta\right), B(t)=\mu(\epsilon-2 a \sinh t)^{-1}$ and set $\alpha=-\ln (1+(\beta+2 \mu) / a)$. It is easy to see that the coefficient $A(t)$ decreases in $t$, $t<0$, whereas $B(t)$ is an increasing function and for $t<\alpha$, the inequality $B(t)<A(t)$ holds.

Consider the following domains in the plane $(s, t)$ :

$$
\begin{gathered}
D_{1}=\{(s, t) \in D: s \leq t \leq \alpha\}, \\
D_{2}=\{(s, t) \in D: t \geq s \geq \alpha\}, \\
D_{3}=\{(s, t) \in D: t \geq \alpha, s \leq \alpha\} .
\end{gathered}
$$

Obviously, $D=D_{1} \cup D_{2} \cup D_{3}$ and it is enough to prove the claim in each of domains $D_{1}, D_{2}$ and $D_{3}$. Since $D_{2}$ is a compact set and $U(t, s)$ is a continuous function, the proof is non-trivial only in $D_{1}$ and $D_{3}$.

Consider the following subdomains of $D_{1}: D_{1}^{n}=\left\{(s, t) \in D_{1}: t \leq \alpha-1 / n\right\}$, $n=1,2, \ldots$. Since the closure of the set $\bigcup_{n>1} D_{1}^{n}$ coincides with $D_{1}$, it follows from the continuity of function $U(t, s)$ that it is sufficient to prove its boundedness in each of domains $D_{1}^{n}, n=1,2, \ldots$ If $t \leq \alpha-1 / n$ then $A(t) \geq A(\alpha-1 / n)$, $\sup _{t \leq \alpha-1 / n}$ $|B(t)| \leq B(\alpha-1 / n)<A(\alpha-1 / n)$ and therefore, according to Hale ([4], equation (5.9.2)), the trivial solution of equation (3.11) is uniformly stable for $t \leq \alpha-1 / n$. This means that for any $\sigma \leq \alpha-1 / n$ and $d>0$ there exists $\delta=\delta(d)$ such that the inequality $\|\varphi\|<\delta$ implies $\left\|\left(f_{2}\right)_{t}(\sigma, \varphi)\right\|<d$ for all $\sigma \leq t \leq \alpha-1 / n$. Here $\|\cdot\|$ denotes a sup-norm and the function $f_{2}(\sigma, \varphi)$ is the same as in Theorem 3.1.

Let us now represent equation (3.11) in operator form. That is

$$
f^{\prime}(t)=L\left(t, f_{1}(\cdot)\right)
$$

where $L(t, \varphi), t \leq 0$ and $\varphi \in C([-\gamma, 0])$ is the following operator:

$$
L(t, \sigma)=A(t) \varphi(0)-B(t) \varphi(-\gamma) .
$$

Since for $t<\alpha-1 / n$ both $A(t)$ and $B(t)$ are between 0 and 1 ,

$$
|L(t, \varphi)| \leq m\|\varphi\|, \quad m=2 \text {. }
$$

Applying Hale ([4], Lemma 6.6.2) to equation (3.11) and making use of (3.12), we obtain the claim in $D_{1}^{n}$.

For any fixed $s<\alpha$, the function $U(t, s)$ satisfies the following equation 


$$
f(t)=\int_{\alpha}^{t} L\left(u, f_{u}(\cdot)\right) d u+U(\alpha, s), t \in[\alpha, 0]
$$

with an initial condition $f_{\alpha}(\cdot)=U_{\alpha}(\cdot, s)$. Let us note that if $t<0$, then (3.12) also holds with $m=(2 \mu+\beta) / \epsilon$. Since $(s, \alpha) \in D_{1}$ it follows from (3.12) and (3.13) that

$$
\begin{gathered}
|U(t, s)| \leq \int_{\alpha}^{t} \mid L\left(u, U_{u}(\cdot, s)|d u+| U(\alpha, s) \mid\right. \\
\leq m \int_{\alpha}^{t}\left\|U_{u}(\cdot, s)\right\| d u+K, \quad K \underset{(t, s) \in D_{1}}{=\sup _{(t, s) \mid .}} \mid U(t,
\end{gathered}
$$

Therefore, the following inequality holds:

$$
\left\|U_{t}(\cdot, s)\right\| \leq m \int_{\alpha}^{t}\left\|U_{u}(\cdot, s)\right\| d u+K .
$$

Applying Gronwall's lemma to (3.14) we get

$$
\left\|U_{t}(\cdot, s)\right\| \leq K_{1}, K_{1}=K e^{m \alpha}, t \in[\alpha, 0] .
$$

Hence, $\sup _{\alpha<t<0}|U(t, s)| \leq K_{1}$. Since the constant $K_{1}$ does not depend on $s$, it follows that $U(\bar{t}, s)$ is bounded in $D_{3}$ as well.

Remark: All results cited in the proof relate to the case when the functionaldifferential equation is given on the whole real line, whereas we consider it in th interval $(-\infty, \alpha-1 / n]$. However, setting in (3.10) $A(t)=A(\alpha-1 / n), B(t)=$ $B(\alpha-1 / n), t>\alpha-1 / n$ we get an equation which is defined on the whole real line and coincides with $(3.10)$ in $(-\infty, \alpha-1 / n]$. Since all cited results hold true for the last equation, it follows that they hold for equation (3.10) in the interval $(-\infty, \alpha-1 / n]$ as well.

\section{The Solution of System (1.1)-(1.3)}

We begin this section with the following lemma.

Lemma 4.1: Let us set $X(\sigma, t)=f_{2}\left(\sigma, 1+\beta / a+e^{-(\cdot)}\right)(t), \sigma \leq-\gamma$, where as in Theorem 3.1, $f_{2}\left(\sigma, 1+\beta / a+e^{-(\cdot)}\right)(t)$ denotes the solution of equation (3.6) with an initial condition $\varphi(t)=1+\beta / a+e^{-t}$ given on $[\sigma-\gamma, \alpha]$. Then the following holds:

$$
\begin{gathered}
X(\sigma, t) \geq 1+\beta / a+e^{-t}, t \in[\sigma, \sigma+\gamma], \\
\max _{t \in[\sigma, \sigma+\gamma]}\left[X(\sigma, t)-1-\beta / a-e^{-t}\right]=O\left(e^{\sigma}\right), \sigma \rightarrow-\infty .
\end{gathered}
$$

Proof: If $t \in[\sigma, \sigma+\gamma]$ then $X(\sigma, t)$ can be calculated by the formula (3.8), where function $U(t, s)$ is given by (3.2). Setting in (3.8), $\varphi(s)=1$ and $\varphi(s)=e^{-s}$ and calculating the corresponding integrals we obtain the functions

$$
K_{1}(t)=U(t, \sigma)+\frac{\mu}{a m}\left[e^{-t}+1+\frac{\lambda+\beta}{1}\right.
$$




$$
\begin{gathered}
\left.-U(t, \sigma)\left(e^{-\sigma}+1+\frac{\lambda+\beta}{a}\right)\right] \\
K_{2}(t)=e^{-t} V(t, \sigma)+\frac{\lambda}{a m}\left\{1+\left(1+\frac{\mu+\beta}{a}\right) e^{-t}\right. \\
\left.-U(t, \sigma)\left[1+\left(1+\frac{\mu+\beta}{a}\right) e^{-\sigma}\right]\right\}
\end{gathered}
$$

where

$$
m=-\left(x_{1}-x_{2}\right)^{2} b c=\left(1+\frac{\mu+\beta}{a}\right)\left(1+\frac{\lambda+\beta}{a}\right)-1,
$$

and both $V(t, \sigma)$ and $U(t, \sigma)$ being given by (3.1) and (3.2), respectively. Calculating the derivative of the function $X(\sigma, t)-1-\beta / a-e^{-t}=(1+\beta / a) K_{1}(t)+K_{2}(t)-$ $1-\beta / a-e^{-t}$ w.r.t. $t$ we obtain the following expression:

$$
\begin{aligned}
-m^{-} & {\left[\frac{\lambda}{a}\left(1+\frac{\mu+\beta}{a}\right)+\frac{\mu}{a}(1+\beta / a)-m\right]\left\{1+\frac{e^{t}[1+(\mu+\beta) / a]-1}{\left(1+x_{1} e^{t}\right)\left(1+x_{2} e^{t}\right)}\right.} \\
& \left.\times\left(\frac{1+x_{2} e^{\sigma}}{1+x_{2} e^{t}}\right)^{b}\left(\frac{1+x_{1} e^{\sigma}}{1+x_{1} e^{t}}\right)^{c}\left[e^{\sigma}(1+(\lambda+\beta) / a)+1\right]\right\} e^{-t} .
\end{aligned}
$$

It is easy to verify that the number $\frac{\lambda}{a}\left(1+\frac{\mu+\beta}{a}\right)+\frac{\mu}{a}(1+\beta / a)$ is less than $m$. Thus, the first factor in this expression is negative. On the other hand, simple calculations show that the second factor increases in $t$. Hence, it reaches its minimum at point $t=\sigma$ and this minimum is equal to $m e^{2 \sigma}\left(1+x_{1} e^{\sigma}\right)^{-1}\left(1+x_{2} e^{\sigma}\right)^{-1}>0$. Therefore, the function $X(\sigma, t)-1-\beta / a-e^{-t}$ increases on $[\sigma, \sigma+\gamma]$ and since $X(\sigma, \sigma)-1-$ $\beta / a-e^{-\sigma}=0$ it follows that $(i)$ holds and that it attains its maximum at point $t=$ $\sigma+\gamma$. Let us set $t=\sigma+\gamma$ in (4.3) and define a new function $\bar{K}_{1}(u)$ so that $\bar{K}_{1}\left(e^{\sigma}\right)=K_{1}(\sigma+\gamma)$. The function $\bar{K}_{1}(u)$ is equal to:

$$
\bar{K}_{1}(u)=\frac{\mu}{a m} \cdot \frac{\mu}{\lambda} R(u)+S(u)
$$

where

$$
\begin{gathered}
R(u)=u^{-1}-u^{-1}\left(\mu \frac{1+x_{2} u}{\mu+\lambda x_{2} u}\right)^{b}\left(\mu \frac{1+x_{1} u}{\mu+\lambda x_{1} u}\right)^{c}, \\
S(u)=\frac{\mu}{\lambda}\left(\mu \frac{1+x_{2} u}{\mu+\lambda x_{2} u}\right)^{b}\left(\mu \frac{1+x_{1} u}{\mu+\lambda x_{1} u}\right)^{c} \\
\times\left[1-\frac{\mu}{a m}\left(1+\frac{\lambda+\beta}{a}\right)\right]+\frac{\mu}{a m}\left(1+\frac{\lambda+\beta}{a}\right) .
\end{gathered}
$$

In a neighborhood of point $u=0$

$$
\left(\mu \frac{1+x_{2} u}{\mu+\lambda x_{2} u}\right)^{b}\left(\mu \frac{1+x_{1} u}{\mu+\lambda x_{1} u}\right)^{c}=1+\frac{\epsilon}{\mu}\left(1+\frac{\lambda+\beta}{a}\right) u+O\left(u^{2}\right)
$$

and hence

$$
R(u)=-\frac{\epsilon}{\mu}\left(1+\frac{\lambda+\beta}{a}\right)+O(u), u \rightarrow 0 .
$$

Taking in (4.8) only the first term of the Taylor's series of the function in the left- 
hand side of (4.9), we get

$$
S(u)=\frac{\mu}{\lambda}\left[1-\frac{\mu}{a m}\left(1+\frac{\lambda+\beta}{a}\right)\right]+\frac{\mu}{a m}\left(1+\frac{\lambda+\beta}{a}\right)+O(u), u \rightarrow 0 .
$$

It follows from (4.6), (4.10), and (4.11) that

$$
\bar{K}_{1}(u)=\mu / \lambda+O(u), u \rightarrow 0 .
$$

Similarly, calculating the value of function $K_{2}(t)-e^{-t}$ at point $t=\sigma+\gamma$ and making the substitution $u=e^{\sigma}$ we obtain:

$$
\bar{K}_{2}(u)-\frac{\mu}{\lambda} u^{-1}=R(u)\left[\frac{\lambda}{a m}\left(u+1+\frac{\mu+\beta}{a}\right)-1\right]+\frac{\epsilon \lambda}{a \mu m},
$$

where function $\bar{K}_{2}(u)$ is defined in such a way that $\bar{K}_{2}\left(e^{\sigma}\right)=K_{2}(\sigma+\gamma)$. Making use of (4.10) we get

$$
\bar{K}_{2}(u)-\frac{\mu}{\lambda} u^{-1}=\frac{\epsilon}{\mu}\left(1+\frac{\beta}{a}\right)+O(u), u \rightarrow 0 .
$$

Since $X(\sigma, t)-1-\beta / a-e^{-t}=K_{2}(t)-e^{-t}+(1+\beta / a)\left[K_{1}(t)-1\right]$ the second claim of the lemma follows from (4.12) and (4.13). line.

Theorem 4.2: The equation (2.1) has a non-trivial solution on the negative half-

Proof: Consider the following functions defined on $(-\infty, 0]$ :

$$
\eta_{n}(t)=\left\{\begin{array}{cc}
1+\beta / a+e^{-t}, & t \leq-n \gamma \\
X(-n \gamma, t), & t \in[-n \gamma, 0] .
\end{array}\right.
$$

We shall prove that the sequence $\left\{\eta_{n}\right\}, n \geq 1$ converges uniformly to some function $\eta(t)$ which satisfies equation (2.1) on the whole negative half-line.

Let us consider the series

$$
\eta_{1}(t)+\sum_{n=1}^{\infty}\left[\eta_{n+1}(t)-\eta_{n}(t)\right]
$$

To find the function $\eta_{n+1}(t)$ on $[-n \gamma, 0]$ one must solve equation (2.1) with an initial condition $X(-(n+1) \gamma, t), t \in[-(n+1) \gamma,-n \gamma]$. Hence, making use of formula (3.8) we obtain:

$$
\left\{\begin{array}{cc}
\eta_{n+1}(t)-\eta_{n}(t)= & t \leq-(n+1) \gamma \\
X(t)-1-\beta / a-e^{-t}, & t \in[-(n+1) \gamma,-n \gamma] \\
{\left[X(-n \gamma)-1-\beta / a-e^{n \gamma}\right] U(t,-n \gamma)} & \\
\left.-\mu \int=-n \gamma+n+1\right) \gamma\left[X(s)-1-\beta / a-e^{-s}\right] & t \in[-n \gamma, 0],
\end{array}\right.
$$


where, for the sake of brevity, we have omitted the first argument of the function $X(-(n+1) \gamma, t)$. Therefore, it follows from Theorem 3.2, Lemma 4.1, and the convergence of the integral $\int_{-\infty}^{0}(\epsilon-2 a \sinh s)^{-1} d s$ that for all sufficiently large $n$,

$$
\left|\eta_{n+1}(t)-\eta_{n}(t)\right| \leq K e^{-n \gamma}, K=\text { const. }
$$

Thus, the series (4.14) converges uniformly to some function $\eta(t)$ on $(-\infty, 0]$.

It remains to be proved that this function satisfies equation $(2.1)$ on $(-\infty, 0]$.

Let $t \in[-\gamma, 0]$. Since for every $n \geq 1$ the function $\eta_{n}(t)$ satisfies equation $(2.1)$ on $[-\gamma, 0]$, it follows that

$$
\eta_{n}^{\prime}=-A(t) \eta_{n}(t)-B(t) \eta_{n}(-\gamma), n=1,2, \ldots
$$

where $A(t)$ and $B(t)$ are the coefficients of equation (2.1) defined after formula (3.11). Utilizing (4.15) we easily deduce that

$$
\begin{gathered}
\eta_{1}^{\prime}(t)+\sum_{n=1}^{\infty}\left[\eta_{n+1}^{\prime}(t)-\eta_{n}^{\prime}(t)\right]=-A(t)\left\{\eta_{1}(t)+\sum_{n=1}^{\infty}\left[\eta_{n+1}(t)-\eta_{n}(t)\right]\right\} \\
-B(t)\left\{\eta_{1}(t-\gamma)+\sum_{n=1}^{\infty}\left[\eta_{n+1}(t-\gamma)-\eta_{n}(t-\gamma)\right]\right\} \\
=-A(t) \eta(t)-B(t) \eta(t-\gamma)
\end{gathered}
$$

Therefore, differentiating series (4.14), we obtain that in this case, the function $\eta(t)$ satisfies equation (2.1).

If $t \in[-(k+1) \gamma,-k \gamma], k \geq 1$, then we can represent function $\eta(t)$ in the form

$$
\eta(t)=\eta_{k+1}(t)+\sum_{n=k+1}^{\infty}\left[\eta_{n+1}(t)-\eta_{n}(t)\right]
$$

and repeat the same considerations taking in (4.15) $n=k+1, k+2, \ldots$

Now we are able to characterize the set of all solutions of equation (1.2) on the negative half-line.

Theorem 4.3: The function

$$
f_{2}(t)=-\frac{1+\beta / a+e^{-t}}{\beta(2+\beta / a)}+C \eta(t), C=\text { const. }
$$

is a general solution of equation (1.2) on $(-\infty, 0]$.

Proof: According to Theorems 2.2 and 4.2 function $f_{2}(t)$ is a solution of equation (1.2) for all $C$. It remains to be proved that every solution of this equation can be represented in the form (4.16) with some constant $C$. Let $f(t)$ be such a solution. Then, Theorem 2.1 (ii) implies that it has the asymptotic $C_{1}\left(1+\beta / a+e^{-t}\right)+o(1)$ as $t \rightarrow-\infty$ with some constant $C_{1}$. On the other hand, it follows from the proof of Theorem 4.2 that function $\eta(t)$ has the asymptotic $1+\beta / a+e^{-t}+o(1)$ as $t \rightarrow-\infty$. Therefore, taking in (4.16) $C=C_{1}+\beta^{-1}(2+\beta / a)^{-1}$ we obtain a solution $f_{2}(t)$ that has the same asymptotic as $t \rightarrow-\infty$. Thus, for every $\delta>0$ there exists $\sigma<0$ such that

$$
\sup _{t \leq \sigma}\left|f(t)-f_{s}(t)\right|<\delta
$$


If $t \in[\sigma, 0]$ then, in view of Hale ([4], Theorem 6.3.2), both functions $f(t)$ and $f_{2}(t)$ have the following representation:

$$
\begin{aligned}
& f(t)=f(\sigma) U(t, \sigma)-\mu \int_{\sigma-\gamma}^{\sigma} f(s) U(t, s+\gamma)[\epsilon-2 a \sinh (s+\gamma)]^{-1} d s \\
& +\int_{\sigma}^{t} U(t, s)(\epsilon-2 a \sinh s)^{-1} d s \\
& f_{2}(t)=f_{2}(\sigma) U(t, \sigma)-\mu \int_{\sigma-\gamma}^{\sigma} f_{2}(s) U(t, s+\gamma)[\epsilon-2 a \sinh (s+\gamma)]^{-1} d s \\
& +\int_{\sigma}^{t} U(t, s)(\epsilon-2 a \sinh s)^{-1} d s .
\end{aligned}
$$

Hence,

$$
\begin{gathered}
f(t)-f_{2}(t)=\left[f(\sigma)-f_{2}(\sigma)\right] U(t, \sigma) \\
-\mu \int_{\sigma-\gamma}^{\sigma}\left[f(s)-f_{2}(s)\right] U(t, s+\gamma)[\epsilon-2 a \sinh (s+\gamma)]^{-1} d s
\end{gathered}
$$

and utilizing (4.17) and the boundedness of function $U(t, s)$ we get

$$
\max _{t \in[\sigma, 0]}\left|f(t)-f_{2}(t)\right|<K \delta, K=\text { const. }
$$

Since the number $\delta>0$ in (4.17) and (4.18) is arbitrary, it follows that $f(t)=f_{2}(t)$.

Utilizing the last theorem we are in a position to prove our main result about the solution of system (1.1)-(1.3).

Theorem 4.4: System (1.1)-(1.3) has a unique solution.

Proof: According to Theorem 4.3, the general solution of equation (1.2) on $(-\infty, 0]$ is given by formula (4.16). To satisfy (1.1) and (1.3) on must multiply (4.16) by $e^{t}$ on $[-\gamma, 0]$ and solve equation (1.1) with an initial condition $e^{t} f_{2}(t)$, $t \in[-\gamma, 0]$. We shall show that, taking a suitable constant $C$ in (4.16), we can escape the singularity that equation (1.1) has at point $t=\ln x_{1}$. Then, since the coefficients of this equation are unbounded only in a neighborhood of this point, the existence and uniqueness of its solution will follow from the well-known result from the theory of linear functional-differential equations (Hale [4], Theorem 6.1.1).

Let us set $k=\ln x_{1}$ and take an arbitrary real $\delta \in(0, k \wedge(\gamma / 2))$. Then, it follows from the choice of $\delta$ and formula (3.1) that the solution of equation (1.1) has the following representation in the interval $[k-\delta, k+\delta]$ :

$$
\begin{gathered}
f_{1}(t)=V(t, k-\delta) f_{1}(k-\delta) \\
-\lambda \int_{k-\delta-\gamma}^{k-\delta} f_{1}(s)[\epsilon-2 a \sinh (s+\gamma)]^{-1} V(t, s+\gamma) d s
\end{gathered}
$$




$$
\begin{gathered}
+\int_{k=\delta}^{t}(\epsilon-2 a \sinh s)^{-1} V(t, s) d s \\
=\left(1+x_{2} e^{t}\right)^{-b}\left(1+x_{1} e^{t}\right)^{-c}\left\{\left(1+x_{2} e^{k-\delta}\right)^{b}\left(1+x_{1} e^{k-\delta}\right)^{c} f_{1}(k-\delta)\right. \\
\left.+\int_{k-\delta}^{t}\left[1-\lambda f_{1}(s-\gamma)\right](\epsilon-2 a \sinh s)^{-}\left(1+x_{2} e^{s}\right)^{b}\left(1+x_{1} e^{s}\right)^{c} d s\right\} .
\end{gathered}
$$

Since $1+x_{2} e^{k}=0$ and $b>0$, the function $f_{1}(t)$ will be bounded in a neighborhood of point $t=k$ only if

$$
+\int_{k-\delta}^{k}\left[1=\lambda f_{1}(s-\gamma)\right](\epsilon-2 a \sinh s)^{-1}\left(1+x_{2} e^{s}\right)^{b}\left(1+x_{1} e^{s}\right)^{c} d s=0 .
$$

Making use of L'Hopital's rule, it is easy to verify that condition (4.20) is also sufficient for boundedness of $f_{1}(t)$. On the other hand, Theorem 4.3 implies that

$$
f_{1}(t)=f_{1}^{(1)}(t)+f_{1}^{(2)}(t)+C f_{1}^{(3)}(t), C=\text { const }, t \in[k-\delta-\gamma, k-\delta],
$$

where $f_{1}^{(1)}(t)$ is a solution of equation (1.1) with a zero initial condition and $f_{1}^{(2)}(t)$ and $f_{1}^{(3)}(t)$ are solutions of equation (3.5) with initial conditions on $[-\gamma, 0]$ equal to $-\beta^{-1}(2+(\beta / a))^{-1}\left[1+(1+(\beta / a)) e^{t}\right]$ and $e^{t} \eta(t)$, respectively. Note, that if $t \leq$ $k-\delta$ then the coefficients of both equations (1.1) and (3.5) are bounded and, therefore, all functions in (4.21) are well defined.

Substituting $f_{1}(t)$ from (4.21) into (4.20) we obtain a linear algebraic equation $\widetilde{A} C+\widetilde{B}=0$, for the constant $C$ which always has a solution $C=\bar{C}$ if the coefficient $\widetilde{A}$ is not zero. In this case, taking $C=\bar{C}$ in (4.21), we ensure the fulfillment of condition (4.20) and so we obtain a bounded solution of equation (1.1) in $[k-\delta, k+\delta]$ given by formula (4.19). Since the coefficients of (1.1) are bounded continuous functions provided $t \geq k+\delta$ it follows that this solution can be continued on the whole positive half-line.

Thus, to complete the proof it is enough to show that

$$
\begin{gathered}
\tilde{A}=\left(1+x_{2} e^{k-\delta}\right)^{b}\left(1+x_{1} e^{k-\delta}\right)^{c} f_{1}^{(3)}(k-\delta) \\
-\lambda \int_{k-\delta}^{k} f_{1}^{(3)}(s-\gamma)(\epsilon-2 a \sinh s)^{-1}\left(1+x_{2} e^{s}\right)^{b}\left(1+x_{1} e^{s}\right)^{c} d s \neq 0 .
\end{gathered}
$$

To do this, assume the contrary. Then, repeating for function $f_{1}^{(3)}(t)$ and equation (3.5) the same considerations as those for $f_{1}(t)$ and equation (1.1), we deduce that the function

$$
f_{1}^{(3)}(t)=\left(1+x_{2} e^{t}\right)^{-b}\left(1+x_{1} e^{t}\right)^{-c}\left[\left(1+x_{2} e^{k-\delta}\right)^{b}\left(1+x_{1} e^{k-\delta}\right)^{c} f_{1}^{(3)}(k-\delta)\right.
$$




$$
\left.-\lambda \int_{k-\delta}^{t} f_{1}^{(3)}(s-\gamma)(\epsilon-2 a \sinh s)^{-1}\left(1+x_{2} e^{s}\right)^{b}\left(1+x_{1} e^{s}\right)^{c} d s\right]
$$

remains bounded in the interval $[k-\delta, k+\delta]$. Therefore, $f_{1}^{(3)}$ can be continued on $[k+\delta, \infty)$ so as to satisfy equation (3.5). Hence, in view of Theorems 4.2 and $3.1(i)$, we conclude that the function

$$
\zeta(t)=\left\{\begin{array}{cc}
\eta(t), & t \leq 0 \\
e^{-t} f_{1}^{(3)}(t), & t \geq 0
\end{array}\right.
$$

is a non-trivial global solution of equation (2.1).

Therefore, it follows from Theorem 2.2 that the function $F(t)=f_{2}(t)+\beta(2+$ $\beta / a) \zeta(t), f_{2}(t)$ being given by (2.13), is a global solution of equation (1.2) such that $F(t) \rightarrow 0$, as $t \rightarrow-\infty$. However, from Donchev [3, Theorem 3.1], we obtain that $F(t)$ has a singularity at point $t=\ln x_{1}$. This is a contradiction that proves (4.22).

\section{Acknowledgement}

My thanks are addressed to both the anonymous referees and the associate editor for their constructive remarks.

\section{References}

[1] Davis, M.H.A., On the point process disorder problem, In: Proc. of a Conf. on Math. Control, Zacopane, Poland, PWN, Warsaw (1976), 65-72.

[2] Donchev, D.S., A Problem of Optimal Detection of Jump-Times of a Poisson Process, University of Bonn 1995. (Technical report)

[3] Donchev, D.S., On the two-armed Bandit problem with non-observed Poissonian switching arms, Math. Methods of Operations Research 3 (1998), to appear.

[4] Hale, J, Theory of Functional Differential Equations, Springer-Verlag, New York-Heidelberg-Berlin 1977.

[5] Shiryaev, A.N., Statistical Sequential Analysis, Nauka, Moscow 1979.

[6] Wickwire, K., On the Poisson disorder problem, Int. J. Systems Sci. 10:3 (1979), 275-283. 


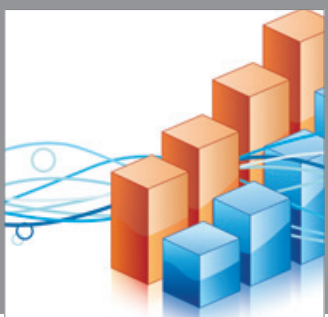

Advances in

Operations Research

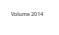

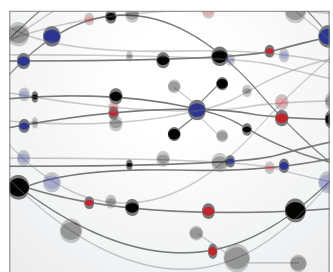

\section{The Scientific} World Journal
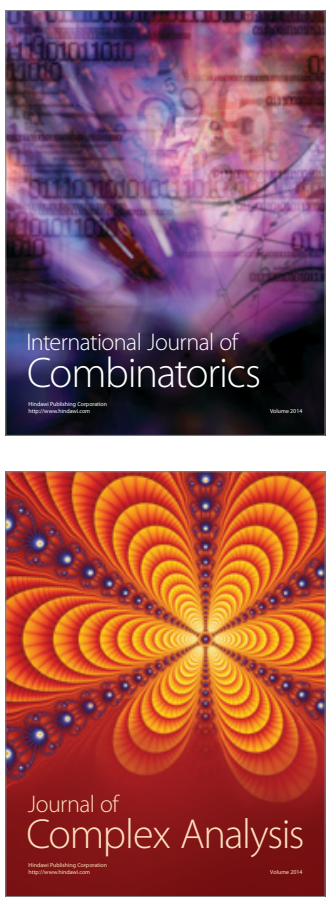

International Journal of

Mathematics and

Mathematical

Sciences
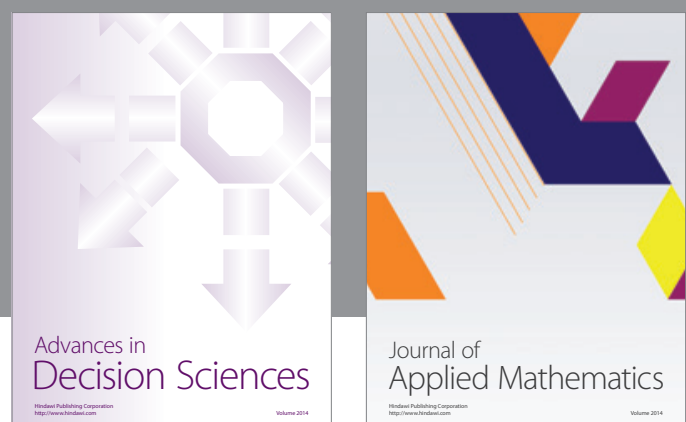

Journal of

Applied Mathematics
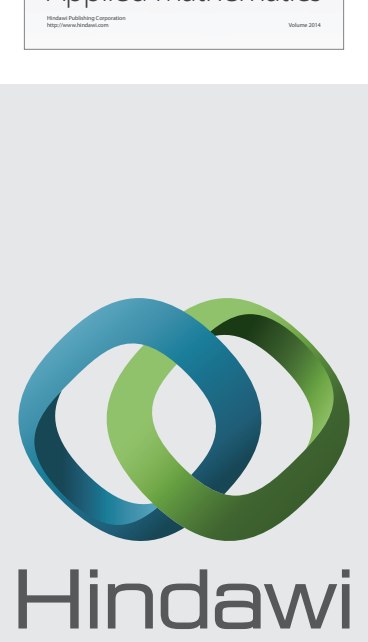

Submit your manuscripts at http://www.hindawi.com
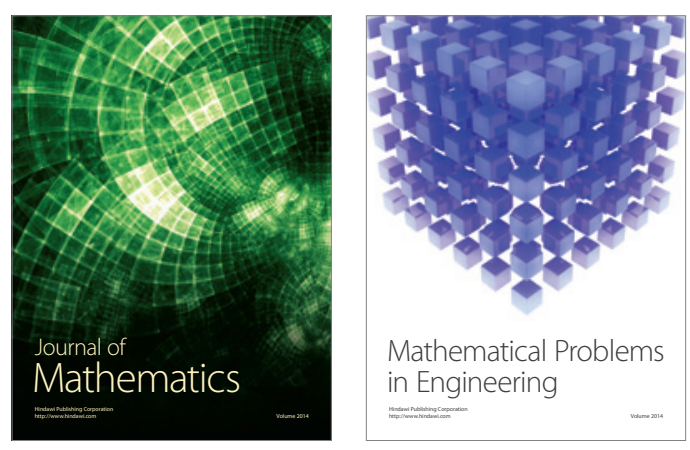

Mathematical Problems in Engineering
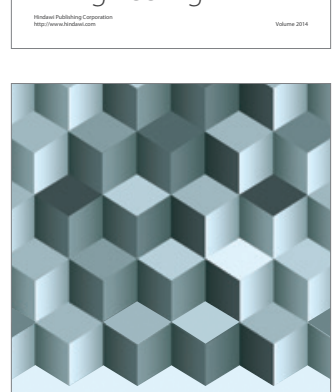

Journal of

Function Spaces
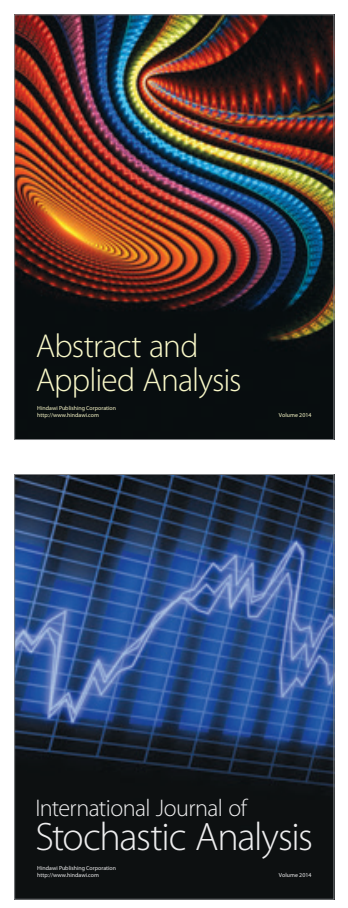

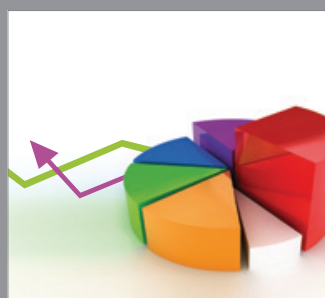

ournal of

Probability and Statistics

Promensencen
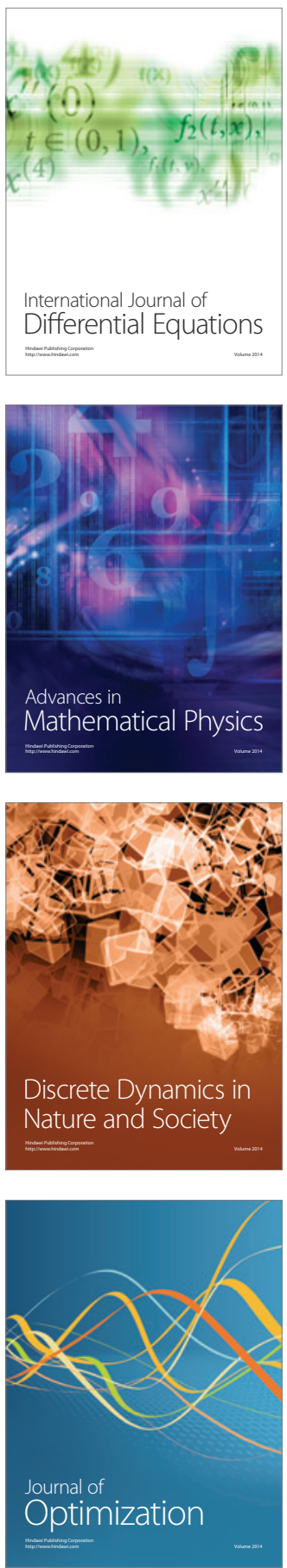